\title{
Acoustic Nearfield and Farfield for Vibrating Piston in Geometrical and Intensity Formulations
}

\author{
M.S. KOZIEŃ* \\ Institute of Applied Mechanics, Cracow University of Technology, al. Jana Pawła II 37, 31-864 Cracow, Poland \\ In classic acoustics there are two areas identified around acoustic sources - nearfield and farfield. The \\ nearfield is connected with the Fresnel solution and farfield - the Fraunhofer one. For each regions there are \\ different theoretical formulae for determination of distribution of the chosen acoustic parameter. Unfortunately \\ there is no sharply outlined border between regions. Therefore one of the important problem, is to define \\ approximately conditions for state the border between them. The two attempts for identification are discussed, \\ i.e. geometrical one and intensity ones. The results are shown on the vibrating circular rigid piston case.
}

PACS: 43.20.Bi, 43.20.Tb, 43.40.Rj

\section{Introduction}

Vibrating surface structural elements are sources of sound radiation. Estimation of the values of acoustic parameters of the generated sound fields is very important problem from engineering point of view. In classic acoustics there are two areas identified around acoustic sources - nearfield and farfield. In experimental vibroacoustics the third area is identified too - very near field. The nearfield is connected with the Fresnel solution and farfield with the Fraunhofer solution. For each region there exist different theoretical formulae for determination of distribution of the chosen acoustic parameter. Unfortunately, there is no sharply outlined border between regions. Therefore one of the important problem is to define approximately conditions for state of the border between them.

The aim of the paper is to compare the values of characteristic quantities, which estimate the position of the region's borders, based on the two interpretations: geometric one and intensity one. The results of sound intensity analytical estimation are compared with thus obtained from experiments. The analyzed structure is vibrating circular rigid piston.

\section{Geometrical interpretation of regions}

The most useful formula describing the acoustic wave propagation in the acoustic medium with existing noise sources is the so-called Kirchhoff integral (1) [8, 13], which gives relationship between values of the acoustic potential $(\varphi)$ for chosen point $P$ inside the acoustic volume, with the values of the same function on the boundary surface, which can include surface type acoustic sources. The formula is not useful, because the unknown function exists on both sides of the relationship

* e-mail: kozien@mech.pk.edu.pl

$$
\varphi(P)=-\frac{1}{4 \pi} \int_{(S)}\left[\varphi \frac{\partial\left(\frac{\mathrm{e}^{-\mathrm{i} k r}}{r}\right)}{\partial n}-\frac{\mathrm{e}^{-\mathrm{i} k r}}{r} \frac{\partial \varphi}{\partial n}\right] \mathrm{d} S,
$$

where $P$ - control point, $\varphi-$ acoustic potential function, $k$ - acoustic wave number, $r$ - distance between control point and element on the boundary surface, $n$ normal direction to the boundary surface.

Based on the Kirchhoff integral the better formula for interpretation, known as the Huyghens-Rayleigh integral (2) [13], can be obtained. This formula can be integrated under assumption that plane, baffled source is radiating in open acoustic field

$$
\varphi(P)=\frac{1}{2 \pi} \int_{(S)} \frac{\mathrm{e}^{-\mathrm{i} k r}}{r} \frac{\partial \varphi}{\partial n} \mathrm{~d} S .
$$

Based on the formula (2), under assumption that distance between observation point $(P)$ and the source is relatively high, the new formula, known as the Fresnel integral (3) can be obtained [13]. It is easy to apply, due to normal surface velocity $v_{\mathrm{n}}$ of planar acoustic source exists in the integral, and pressure in chosen point, as the result of integration. The acoustic region in which the formula can be applied is known as the nearfield of acoustic source. The Cartesian coordinate system is assumed for description of geometry. The origin of coordinates $O$ is the chosen point of the planar source (center of gravity is suggested). The $x$ and $y$ axes are in-plane with the planar source

$$
\begin{aligned}
p & =\frac{\mathrm{i} k \rho c}{2 \pi} \frac{\mathrm{e}^{-\mathrm{i} k r_{0}}}{r_{0}} \int_{(S)} v_{n}(x, y) \exp \left(\mathrm { i } k \left(\left(x \cos \left(r_{0} x\right)\right.\right.\right. \\
& \left.+y \cos \left(r_{0} y\right)\right)+\frac{1}{2 r_{0}}\left(x \cos \left(r_{0} x\right)+y \cos \left(r_{0} y\right)\right)^{2} \\
& \left.\left.-\frac{\rho^{2}}{2 r_{0}}\right)\right) \mathrm{d} S,
\end{aligned}
$$

where $p$ - acoustic pressure in control point, $\rho$ - density of the acoustic medium, $c$ - speed of sound in the acoustic medium, $k$ - acoustic wave number, $v_{\mathrm{n}}-$ normal velocity of the planar acoustic source, $r_{0}-$ dis- 
tance between control point and origin of coordinates $O$, $\rho^{2}=x^{2}+y^{2}$.

Based on the formula (3), under assumption that distance between observation point $(P)$ and the source is big in comparison with the source dimensions (lines connecting observation point with all points of the source are parallel in practice), the new formula, known as the Fraunhofer integral (4) can be obtained [13]. It is easier to apply than the Fresnel integral. The acoustic region in which the formula can be applied is known as the farfield of acoustic source

$$
\begin{aligned}
p & =\frac{\mathrm{i} k \rho c}{2 \pi} \frac{e^{-\mathrm{i} k r_{0}}}{r_{0}} \int_{(S)} v_{n}(x, y) \exp \left(\mathrm { i } k \left(x \cos \left(r_{0} x\right)\right.\right. \\
& \left.\left.+y \cos \left(r_{o} y\right)\right)\right) \mathrm{d} S .
\end{aligned}
$$

Different assumptions define the three characteristic regions in the acoustic surface, around the radiated structure: very near field, nearfield and farfield. The known in literature distances of each of the region's borders are given in Table I.

TABLE I

Geometrical conditions for acoustics regions. $D$ - the biggest characteristic dimension of the source (diameter for circle), $\lambda$ - acoustic wavelength, $r_{0}-$ distance from the source (center of gravity).

\begin{tabular}{c|c|c}
\hline \hline Region & Condition & Literature \\
\hline very near field & $r_{0} \ll \frac{D}{2 \pi} \ll \frac{\lambda}{2 \pi}$ & {$[1]$} \\
(direct field, hydrodynamic zone) & $r_{0} \ll \lambda \vee r_{0}<2 D$ & {$[11,12]$} \\
\hline nearfield & $r_{0} \ll \frac{D}{2 \pi} \ll \frac{\lambda}{2 \pi}$ & {$[1]$} \\
(the Fresnel zone) & $\frac{D^{2}}{r_{0}^{2}} \ll 1 \wedge r_{0} \gg \lambda$ & {$[4,8,11-13]$} \\
\hline & $r_{0} \gg \frac{\lambda}{2 \pi}$ & {$[1]$} \\
farfield & $r_{0} \gg \lambda \vee r_{0} \gg 2 D$ & {$[11,12]$} \\
(the Fraunhofer zone) & $r_{0} \gg 2 \pi \frac{D^{2}}{\lambda}$ & {$[4,8]$} \\
& $r_{0} \gg \frac{\pi}{4} \frac{D^{2}}{\lambda}$ & {$[10,13]$}
\end{tabular}

\section{Intensity interpretation of regions}

The intensity interpretation can be done when the analysis of the sound wave propagation is given in complex variable space [9]. In this case the sound acoustic vector has two components: active (real) one, and reactive (imaginary) one. Time averaged over period component of reactive intensity is equal to zero, hence the active component describes the energy flow. If in the considered region, the value of reactive intensity part is not so small in comparison with the active one, we say that it is the acoustic nearfield region. In the opposite case the region is called as the acoustic farfield one.

It should be noted that the acoustic and geometric nearfields do not define the same regions in the acoustic medium or the same values of the limit distances of the zones. The example of this fact is radiation of a point source [9].

\section{Basis of the hybrid intensity method}

The hybrid intensity method was proposed by the author [5, 6]. Analysis of the acoustic field radiated by vibrating surface structural elements is based on determination of the acoustic intensity vector $\bar{I}$ in chosen acoustic space point. The vibrating elements are divided on sub-areas. If the structural analyses are done by the finite element method, such kind of partition is the element mesh map. Each vibrating element is a source of radiated sound. Let us consider the monochromatic acoustic wave case with angular frequency $\omega$. For acoustic analysis it is assumed that each sub-area is a small surface element [7]. Definition "small" is understood in comparison with the length of the acoustic wave $\lambda$, connected with its angular frequency $\omega$. Analysis is described in Cartesian coordinate system Oxyz. Position of the arbitrary chosen control point $P(x, y, z)$ with respect to position of centre of the $i$-th sub-area $Q_{i}\left(x_{i}, y_{i}, z_{i}\right)$ and position of centre of the $i$-th sub-area $Q_{i}\left(x_{i}, y_{i}, z_{i}\right)$ are defined by vectors $\bar{r}_{i}$ and $\bar{\rho}_{i}$, respectively. In this case it is possible to estimate the acoustic pressure (5) and the components of the particle velocity vector (6), point sources (7), produced by $i$-th sub-area in the control point $P[7]$, where $\rho-$ density of the acoustic medium, $c$ - speed of sound in the acoustic medium, $A_{i}$ - amplitudes of vibration, $\omega-$ angular velocity, $k$ - wave number. The other parameters are schematically shown in Fig. 1.

Superposition of the pressures and velocities comes from all sub-areas in the control point $P$ are done based on the general formula for the case of the system of $N$-th point sources (6) [9]: 


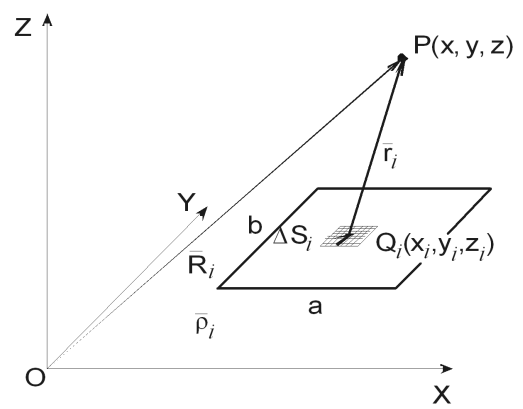

Fig. 1. Geometry of the radiating surface and $i$-th sub-area.

$$
\begin{aligned}
& p_{i}=-\frac{1}{2 \pi} \Delta S_{i} \omega^{2} \rho \frac{A_{i}}{r_{i}} \exp \left(\mathrm{i}\left(\omega t-k r_{i}\right)\right) \\
& \bar{v}_{i}=A_{i} \Delta S_{i}\left(-\frac{\omega^{2}}{2 \pi c r_{i}}+\mathrm{i} \frac{\omega}{2 \pi r_{i}^{2}}\right) \exp \left(\mathrm{i}\left(\omega t-k r_{i}\right)\right) \frac{\bar{r}_{i}}{r_{i}} \\
& \bar{I}=\frac{1}{2} \sum_{j=1}^{\mathrm{n}} p_{j} \cdot \sum_{j=1}^{\mathrm{n}} \bar{v}_{j}^{*} .
\end{aligned}
$$

Based on the formulae (5), (6) and (7), after suitable manipulations, the final relationships for active and reactive parts of the acoustic vector in the control point $P$ can be obtained. The detailed formulae are given in [6]. The formulae make possible to identify the acoustic field type in the chosen point (area) according to the complex variable interpretation.

\section{Determination of acoustic regions for vibrating piston}

Let us consider transversal, harmonically variable vibrations of circular rigid piston with diameter $D$ with constant amplitude of velocity, and given frequency $f$. Based on the hybrid method and knowledge of the parameters of structural vibrations, it is possible to determine the acoustic intensity vector for chosen control points lying on the axis of the piston and distanced $r_{\mathrm{n}}$ from its surface (see Fig. 2).

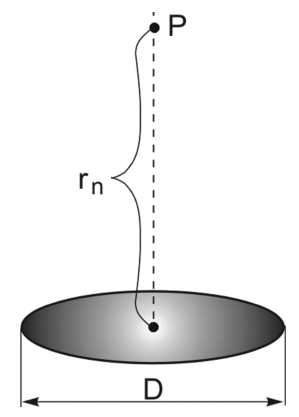

Fig. 2. Geometry of the radiating piston and position of control point.
Values of parameters defining vibrations of the piston are the same as in the experimental test given by Cieślik and Panuszka [2]. The piston with diameter $D=0.14 \mathrm{~m}$ was vibrating with amplitude of velocity equal to $0.05 \mathrm{~m} / \mathrm{s}$ for all considered frequencies. The following frequencies are analysed: 400, 540, 1000, and $1150 \mathrm{~Hz}$.

In Table II there are given characteristic values of distances for the nearfield region for analyzed piston, having in mind formulae given in Table I, for considered values of frequencies. In Table III there are given values of the same parameters for the farfield region.

TABLE II

Distances for the nearfield region for considered values of frequency.

\begin{tabular}{c|c|c|c|c}
\hline \hline \multirow{2}{*}{$f[\mathrm{~Hz}]$} & \multicolumn{3}{|c|}{$r_{0} \gg$} & $r_{0}^{2} \gg$ \\
\cline { 2 - 5 } & $\lambda$ & $\frac{D}{2 \pi}$ & $\frac{\lambda}{2 \pi}$ & $D^{2}$ \\
\hline 400 & 0.85 & 0.02 & 0.14 & 0.02 \\
540 & 0.63 & 0.02 & 0.10 & 0.02 \\
1000 & 0.34 & 0.02 & 0.05 & 0.02 \\
1150 & 0.30 & 0.02 & 0.05 & 0.02
\end{tabular}

TABLE III

Distances for the farfield region for considered values of frequency.

\begin{tabular}{c|c|c|c|c}
\hline \hline \multirow{2}{*}{$f[\mathrm{~Hz}]$} & \multicolumn{4}{|c}{$r_{0} \gg$} \\
\cline { 2 - 5 } & $\frac{1}{4} \frac{D^{2}}{\lambda}$ & $\frac{\pi}{4} \frac{D^{2}}{\lambda}$ & $2 \pi \frac{D^{2}}{\lambda}$ & $2 D$ \\
\hline 400 & 0.006 & 0.018 & 0.15 & 0.28 \\
540 & 0.008 & 0.024 & 0.20 & 0.28 \\
1000 & 0.014 & 0.045 & 0.36 & 0.28 \\
1150 & 0.016 & 0.051 & 0.41 & 0.28
\end{tabular}

The results of analytical estimations and measurements are presented in Tables IV-VII suitable for considered frequencies.

\begin{tabular}{|c|c|c|c|}
\hline \multirow{2}{*}{$r_{\mathrm{n}}[\mathrm{m}]$} & \multicolumn{2}{|c|}{ Hybrid method } & \multirow{2}{*}{$\begin{array}{c}\text { Experiment }[2] \\
I[\mathrm{~dB}]\end{array}$} \\
\hline & $\operatorname{Re}(I)[\mathrm{dB}]$ & $\operatorname{Im}(I)[\mathrm{dB}]$ & \\
\hline 0.1 & 100.9 & 101.7 & 102.5 \\
\hline 0.6 & 86.8 & 80.3 & 85.0 \\
\hline 1.0 & 82.4 & 73.7 & 80.0 \\
\hline 5.0 & 68.4 & 52.8 & - \\
\hline 10.0 & 62.4 & 43.7 & - \\
\hline
\end{tabular}

TABLE IV

Comparison of intensity levels for $f=400 \mathrm{~Hz}$.

The results of analysis show that the experimental results and thus obtained by the hybrid method (the real part) are not far from each other. The differences are not higher than 4-6 dB for all analysed regions.

For the control point not far distanced from the piston area (geometrically very near field and near field), the 
Comparison of intensity levels for $f=540 \mathrm{~Hz}$.

\begin{tabular}{c|c|c|c}
\hline \hline \multirow{2}{*}{$\mathrm{r}_{\mathrm{n}}[\mathrm{m}]$} & \multicolumn{2}{|c|}{ Hybrid method } & Experiment [2] \\
\cline { 2 - 3 } & $\operatorname{Re}(I)[\mathrm{dB}]$ & $\operatorname{Im}(I)[\mathrm{dB}]$ & $I[\mathrm{~dB}]$ \\
\hline 0.1 & 103.5 & 103.0 & 107.5 \\
0.6 & 89.4 & 81.6 & 91.3 \\
1.0 & 85.0 & 75.0 & 87.5 \\
5.0 & 71.0 & 54.1 & - \\
10.0 & 65.0 & 45.0 & -
\end{tabular}

TABLE VI

Comparison of intensity levels for $f=1000 \mathrm{~Hz}$.

\begin{tabular}{c|c|c|c}
\hline \hline \multirow{2}{*}{$\mathrm{r}_{\mathrm{n}}[\mathrm{m}]$} & \multicolumn{2}{|c|}{ Hybrid method } & Experiment [2] \\
\cline { 2 - 3 } & $\operatorname{Re}(I)[\mathrm{dB}]$ & $\operatorname{Im}(I)[\mathrm{dB}]$ & $I[\mathrm{~dB}]$ \\
\hline 0.1 & 108.8 & 105.6 & 112.0 \\
0.6 & 94.8 & 84.3 & 95.6 \\
1.0 & 90.4 & 77.7 & 92.5 \\
5.0 & 76.4 & 56.7 & - \\
10.0 & 70.4 & 47.7 & -
\end{tabular}

values of reactive part are higher or almost equal to the active part. It is the condition of the nearfield in acoustic interpretation.

The values of the reactive part of acoustic intensity vector have significant values in comparison with the active part for higher distances than given by the geometrical condition for the far field. These results are in accordance with conclusions coming from the experimental investigations given in $[11,12]$.

\section{Conclusions}

The results of analysis show that the experimental results and thus obtained by the hybrid method are not far from each other.

The experimental results of the sound radiation by the surface elements given by Weyna $[11,12]$ show that, often, character of the acoustic field in the farfield areas in geometrical meaning is nonlinear. Thus, the geometrical type condition for the farfield is not enough good.

The complex variable analysis of the acoustic field combined with the hybrid method, is the useful practical
Comparison of intensity levels for $f=1150 \mathrm{~Hz}$.

\begin{tabular}{c|c|c|c}
\hline \hline \multirow{2}{*}{$\mathrm{r}_{\mathrm{n}}[\mathrm{m}]$} & \multicolumn{2}{|c|}{ Hybrid method } & Experiment [2] \\
\cline { 2 - 3 } & $\operatorname{Re}(I)[\mathrm{dB}]$ & $\operatorname{Im}(I)[\mathrm{dB}]$ & $I[\mathrm{~dB}]$ \\
\hline 0.1 & 110.0 & 106.1 & 106.3 \\
0.6 & 96.0 & 84.9 & 90.5 \\
1.0 & 91.6 & 78.3 & 86.3 \\
5.0 & 77.6 & 57.3 & - \\
10.0 & 71.6 & 48.3 & -
\end{tabular}

method to analyze the acoustic radiation by vibrating surface elements. The method can be applied to estimation of the type of acoustic field, having in mind definition that in the farfield the analyzed system should be linear.

\section{References}

[1] H.-E. De Bree, V.B. Svetovoy, R. Raangs, R. Visser, in: Proc. ICSV11, 2004.

[2] J. Cieślik, R. Panuszka, Arch. Acoust. 18, 197 (1993).

[3] L. Cremer, M. Heckl, E. Ungar, Structure Borne Sound, Springer-Verlag, Berlin 1988.

[4] Z. Engel, Protection of Environment against Vibrations and Noise, PWN, Warszawa 2001 (in Polish).

[5] M.S. Kozień, J. Theoret. Appl. Mech. 43, 119 (2005).

[6] M.S. Kozień, J. Theoret. Appl. Mech. 47, 411 (2009).

[7] M. Kwiek, Laboratory Acoustics. Part I: Basis Theoretical Acoustics, PWN, Warszawa 1968 (in Polish).

[8] I. Malecki, Theory of Waves and Acoustic Systems, PWN, Warszawa 1964 (in Polish).

[9] J.A. Mann, III, J. Tichy, A.J. Romano, J. Acoust. Soc. Am. 82, 17 (1987).

[10] A. Śliwiński, Ultrasounds and their Applications, WNT, Warszawa 2001 (in Polish).

[11] S. Weyna, Surface Distribution of Acoustic Intensity Vector in Small Limited Volume, Szczecin University of Technology, Szczecin 1993 (in Polish).

[12] S. Weyna, Propagation of Acoustic Energy of Realistic Sources, WNT, Warszawa 2005 (in Polish).

[13] R. Wyrzykowski, Linear Theory of Acoustic Field of Gas Medium, Rzeszów Society of Science Friends, Rzeszów 1970 (in Polish). 